E-Review Dossier 1-2013

Bologna (BraDypuS) 2014

La crisi dei partiti in Emilia Romagna negli anni '70/"80 a cura di Mirco Carrattieri e Carlo De Maria

ISSN: 2282-4979

ISBN: 978-88-98392-05-6 DOI: 10.12977/ereview23 pp. $11-20$

\title{
Introduzione. Un punto di vista sulla crisi
}

La crisi della cosiddetta "prima Repubblica" è ormai un oggetto di ricerca storica. Cominciano a definirsi periodizzazione e eziologia. Le spiegazioni si concentrano per lo più sulla degenerazione del sistema partitico dopo gli anni Settanta. Guardare al fenomeno dal punto di vista emiliano romagnolo può essere utile, sia per verificare le principali tesi storiografiche, sia per evidenziare l'articolazione locale della crisi, tanto più considerando la peculiare subcultura politica della regione.

The crisis of the so-called "First Republic" is today an object of historical research. Scientists are beginning to define periodization and etiology of this crisis. The explanations are focused mostly on the degeneration of the party system after the seventies. Looking at the phenomenon from the point of view of Emilia Romagna may be useful, either to verify the main historiographical thesis, both to highlight the local articulation of the crisis, especially considering the peculiar subculture policy of this region.

\section{I tempi della crisi}

A oltre 20 anni di distanza dal 1992, gli studi sulla crisi del sistema politico dell'Italia repubblicana cominciano a consolidarsi e a delineare una linea storiografica, che supera ormai la mera cronaca e l'ottica della crisi [Giovagnoli 1998], affiancandosi credibilmente e utilmente incrociando gli studi politologici [Morlino e Tarchi (eds.) 2006]. Cominciano così a definirsi la cronologia e l'eziologia del processo.

Per la maggior parte delle ricostruzioni, la parabola del dopoguerra italiano rag- 
giunge il suo apice nei primi anni Settanta [Baldissara (ed.) 2001]. È proprio in questo decennio però, che, fallito il processo riformatore [Tranfaglia 1994] e sperimentate ormai tutte le possibili combinazioni di governo [Craveri 1995], si avvia anche la fase discendente, variamente descritta come snervamento [Ginsborg 1998], logoramento [Scoppola 1991], declino [Mammarella 1993], erosione [Santarelli 1996], crepuscolo [Lupo 1994], corrosione [Crainz 2012].

In particolare il caso Moro del 1978 viene visto come il tornante decisivo, «tragedia repubblicana» [Giovagnoli 2005], che si conclude con un vero e proprio «funerale della Repubblica» [Craveri 2004]. Il sistema dei partiti comincia visibilmente a scricchiolare, come dimostra l'esito del referendum sul finanziamento pubblico di quello stesso anno, nel quale, anche se prevalgono i no, l'alta percentuale di voti favorevoli all'abrogazione segnala una generale disaffezione nei confronti dei partiti.

Il paese, sfiancato dalle difficoltà economiche, precipita in una crisi profonda, accentuata dalla minaccia dell'eversione, vera e propria «notte della Repubblica» [Zavoli 1992]. Alla "crisi delle ideologie" corrisponde un sempre più evidente "riflusso nel privato", che la ripresa trasforma in trionfo del rampantismo [Galli della Loggia 1980]. L'apparente stabilità del pentapartito rappresenta solo una facciata, ma sotto l'arroccamento centrista si consumano i litigi interni a un sistema di potere ormai autoreferenziale, nel quale all'imperfezione del bipartitismo si associa ora l'uso spregiudicato del potere di coalizione da parte del Psi. Si delinea una "alternanza senza reale alternativa"; e le elezioni politiche si svuotano di significato, come dimostra l'aumento dell'astensionismo [Fruncillo 2004]. Inizia «la grande depressione della politica italiana» [Mastropaolo 1996].

La classe dirigente, sempre più consapevole della necessità di riforma, ma sufficientemente proterva da ritenerla sempre procrastinabile (e da sentirsi titolare di una indiscriminata impunità), «galleggia su un consenso drogato da un benessere senza futuro» [Scoppola 1991]. Gli anni Ottanta sono dunque «il decennio lungo del cambiamento frustrato» [Quagliariello 2004]; ma la delusione per le mancate riforme, emblematicamente sintetizzata dal fallimento del progetto demitiano e dall'avvento del "Caf", determinano una drammatica crisi di credibilità e quindi di legittimità del sistema.

Essa risulta «drammatica e profonda» [Ginsborg 1998] e si rivela irreversibile dopo il 1989, quando al crollo del muro di Berlino seguono nel nostro paese la stagione referendaria, Tangentopoli e gli attentati di mafia, che segnano uno sgretolamento progressivo dello Stato. In corrispondenza del terremoto elettorale del 1992, vero e proprio «annus horribilis» [Mammarella 1993], e poi della svolta imposta dal referendum del 1993 («un nuovo 18 aprile» [Lupo 2013]), si avverte 
la frana del sistema, una vera e propria bufera, il tracollo delle istituzioni [Crainz 2012].

Si avvia quindi un travagliato periodo di transizione, di cui però non è semplice identificare la fine: secondo alcuni da porre già nel 1994, quando con la nuova legge elettorale, l'avvento di Berlusconi e il drastico ricambio del ceto parlamentare si ridisegna completamente il sistema partitico; secondo altri solo nel 1996, quando per la prima volta nella storia repubblicana la sinistra assume una piena responsabilità di governo; o magari nel 2001, quando sembra stabilizzarsi un'alternanza di governo [Bartolini e D'Alimonte (eds.) 2002]. Quella italiana più che una rivoluzione appare però «un passaggio d'epoca senza bussola» [Asor Rosa 1996], «una simulazione di guerra civile» [Deaglio 2009]. La transizione appare eternamente incompiuta [Ceccanti e Vassallo (eds.) 2004] e il paese è ancora «alla ricerca di se stesso» [Caracciolo 1999]. La discussa etichetta di "seconda Repubblica", impropria dal punto di vista della forma costituzionale, si impone nel dibattito giornalistico [Zavoli 1999] e filtra anche nella storiografia, nella misura in cui evidenzia il venire meno di una stagione (il cinquantennio repubblicano [Neppi Modona (ed.) 1996]) e dei suoi principali protagonisti [Lupo 2013]. Non pochi osservatori peraltro si interrogano sugli elementi caratterizzanti della nuova fase, che per lo più riconducono alle tentazioni carismatiche e populistiche portate dalla videopolitica berlusconiana [Tranfaglia 2009]. Resta la sensazione che il bipolarismo "all'italiana" riproduca e anzi esasperi vecchi mali, come la demonizzazione dell'avversario [Ventrone (ed.) 2006] o l'inconcludenza decisionale [Gervasoni 2010]. E che al mutare del ceto politico, non abbia corrisposto un reale avvicendamento della classe dominante [Losurdo 1994]. Secondo alcuni si profilerebbe già all'orizzonte una "terza Repubblica", deriva del fallimento della seconda e nostalgicamente legata alla prima [Calise 2006]. In ogni caso è evidente come all'euforia palingenetica del 1992 si sia ormai sostituita un'amara rassegnazione; e come l'Italia rimanga assai distante dalla "repubblica dei cittadini" e dalla "democrazia compiuta" che allora si auspicava.

\section{Alla ricerca delle cause}

Se i tempi della crisi appaiono abbastanza definiti, più complessa è l'identificazione delle sue cause. Essa rimanda infatti a una galassia di fattori, la cui diversa profondità storica e configurazione gerarchica definisce proposte storiografiche anche molto diverse. Per comodità espositiva possiamo ricondurre le spiegazioni avanzate a tre grandi categorie.

Alcune ricostruzioni inseriscono anche questa "crisi di regime" nei tempi lunghi 
dell'Italia unita, recuperando fattori di debolezza strutturali che, lungi dall'essere risolti, nel contesto repubblicano si aggraverebbero ulteriormente. Tra questi si possono ricordare soprattutto lo scarso senso dello stato e delle istituzioni [Pezzino 2002]; la debole identità nazionale [Galli della Loggia 1998]; l'atavico familismo [Tullio-Altan 1986]. Cioè, in sostanza, l'endemico particolarismo, per giunta rafforzato dall'esclusivismo ideologico delle culture politiche postbelliche. Ma si può aggiungere anche il perdurare di sistemi bloccati, che impedisce una reale alternanza di governo, proponendo solo alternative di sistema [Salvadori 1994] o tentazioni trasformistiche [Sabbatucci 2003].

Nella migliore delle ipotesi dunque il 1992 viene inserito nel novero delle "tragedie necessarie" che spingono gli italiani, quando sono sull'orlo del baratro, a reagire dando il meglio di sé e sperimentando la loro creatività anche sul piano istituzionale. Nella peggiore si apre invece la strada all'ennesima discontinuità superficiale e rivoluzione passiva di un paese sostanzialmente incapace di essere "normale" senza versare lacrime e sangue [Lanaro 1992].

Più dense e perspicue appaiono le analisi che concentrano l'attenzione sul dopoguerra, in genere caratterizzando quella italiana come una "repubblica dei partiti" capace sì di portare per la prima volta il paese alla democrazia, ma a una democrazia «speciale», «difficile» [Barbagallo 2009], «protetta», «bloccata», «incompiuta» [Craveri 2002]; intrinsecamente fragile proprio a causa dello strapotere dei partiti, da cui la fortuna del termine "partitocrazia". I partiti, favoriti da un sistema istituzionale troppo bizantino e dal proporzionalismo elettorale, avrebbero di fatto occupato le istituzioni e la società. Fenomeno che la storiografia "revisionista", da De Felice [1995] a Galli della Loggia [1996], fa rimontare alle origini postbelliche del sistema; e che invece secondo altri, da Colarizi [1994] a Ridolfi [2010], si aggrava drasticamente a partire dagli anni Settanta, «primo cambio di fase» [Santarelli 1996] e vero e proprio tornante [Barbagallo 2009] della storia repubblicana.

Mentre infatti nel periodo precedente l'attore principale dell'invasione e appropriazione delle istituzioni sarebbe la Democrazia cristiana (vero e proprio partitoStato) a partire dagli anni Settanta anche il Pci sarebbe direttamente coinvolto, prima a livello di enti locali, poi di parlamento e infine, con il "compromesso storico", persino del governo. Secondo questa visione, anche l'innegabile asimmetria tra un'eterna maggioranza e un'opposizione mai legittimata (che la si definisca "bipartitismo imperfetto" o "pluralismo polarizzato" poco cambia) apparirebbe dunque secondaria rispetto alle forme di scambio occulto (consociativismo) e al prevalere di logiche spartitorie (lottizzazione). Alla responsabilità dei partiti andrebbero quindi ricondotte le principali componenti della "grande 
slavina" che investe il paese all'inizio degli anni Novanta [Cafagna 1993].

Lo stato soffrirebbe infatti di una crisi fiscale dovuta all'uso scriteriato del debito come mezzo per finanziare la spesa pubblica senza sacrificare il consenso elettorale (nel 1991 il debito pubblico supera il Pil); di una crisi morale causata dalla corruzione diffusa, quando non dalle collusioni con progetti e organizzazioni criminali (nel 1992 scoppia "mani pulite" e nel 1993 finisce sotto processo per mafia il senatore e più volte Presidente del Consiglio Giulio Andreotti); di una crisi istituzionale conseguente alla perdita di rendimento dell'azione parlamentare, al proliferare delle "leggine", al trionfo della decretazione d'urgenza (tutti mali che il sistema, nonostante la retorica della riforma e le numerose bicamerali, non sembra in grado di risanare dall'interno, finendo poi per soccombere sotto le picconate di Cossiga, i referendum di Segni, le arringhe di Di Pietro, i governi tecnici di Amato e Ciampi). In ogni caso, alla fine la Repubblica sarebbe implosa dall'interno a causa delle disfunzionalità di un sistema partitico «inefficiente, corrotto, immobile» [Colarizi 2007]; e di un ceto politico «arrogante, impunito e impunibile» [Salvati 2000].

Un terzo e ultimo blocco di studi pone maggiore attenzione ai fattori esogeni al sistema dei partiti, che ne condizionerebbero l'azione e ne indurrebbero per molti aspetti la degenerazione. Si evidenziano in questo senso i vincoli esterni posti dalla situazione internazionale, a partire dal contesto della Guerra fredda e dagli indubbi elementi di etero-direzione che riguardano i due principali partiti sulla scena [Di Nolfo 1996]. In un sistema per il quale "doppio Stato" e "doppiezza" sono credibili categorie interpretative [De Felice 2003], il crollo del socialismo reale in Europa orientale del 1989-91 appare un fattore di destabilizzazione decisivo, a cui vanno aggiunti gli effetti della guerra del Golfo, del trattato di Maastricht e delle nuove migrazioni trans-mediterranee; e più in generale le dinamiche della globalizzazione [Gentiloni Silveri 2008].

Non meno rilevante appare poi la presenza attiva di altre forze quanto mai ingombranti: da quelle legali, come la Chiesa, la classe imprenditoriale o il "quarto potere" dei media; a quelle opache, sotterranee, dai servizi deviati alle logge massoniche; fino a contropoteri dichiaratamente illegali, come le diverse forme di mafia e terrorismo [Flores d'Arcais 2011]. Infine bisogna tenere presenti i nuovi attori politici che, nel corso dell'età repubblicana, intervengono sulla scena politica originariamente dominata dai partiti di massa: quelli post-ideologici, dai radicali ai verdi; i movimenti collettivi, dagli anni Sessanta in poi; i gruppi extraparlamentari, fino alle forme di lotta armata; le lobbies tecnocratiche; le leghe localistiche; le diverse voci dell'antipolitica [Lupo 2013]. Né mancano analisi che mettono in discussione le letture esclusivamente politiche della crisi, chia- 
mando in causa piuttosto le dinamiche proprie di una modernizzazione tardiva, costruita su basi fragili e con metodi poco ortodossi [Sylos Labini 1995], tanto da risultare drammaticamente squilibrata o socialmente insostenibile [Casula (ed.) 1999]. O il nuovo contesto culturale veicolato dalla televisione, in particolare quella commerciale, che ha trasformato i cittadini in consumatori [Gozzini 2011]; e ha riconfigurato il loro rapporto con la memoria [De Luna 2011]. O ancora le trasformazioni nel costume, con una secolarizzazione che troppo spesso ha significato anche caduta della tensione morale e prevalere di un tecnicismo cinico e spregiudicato [Berselli 2003].

Ove da un lato occorre precisare come, a valle e a monte di questi processi, si ritrovino comunque le responsabilità della politica; e dall'altra però si deve, sulla scia di Pasolini, mettere in discussione la sempre facile dicotomia tra società politica e società civile (o paese legale e paese reale, o palazzo e piazza, ecc.), che in più circostanze gli italiani hanno evocato a propria consolatoria giustificazione, facendo della "casta" il capro espiatorio delle difficoltà, senza considerare che in un paese democratico essa riflette le scelte degli elettori e non di rado anche i loro vizi. Il tutto tenendo conto del fatto che di "crisi della democrazia" di fronte alla società postindustriale e alla cultura postmoderna si parla in tutto l'Occidente, con caratteri comuni che l'indubbia specificità italiana non può oscurare.

\section{Vista dall'Emilia Romagna}

Il presente dossier parte dal presupposto che uno studio della crisi del sistema politico italiano dal punto di vista regionale dell'Emilia Romagna possa fornire un contributo euristico significativo alle tematiche sin qui accennate.

Innanzitutto perché l'analisi dei contesti locali permette di riconfigurare e spesso precisare il quadro analitico, verificando nel concreto della dinamica storica l'interazione e la gerarchia tra i vari fattori evidenziati. Aggiungo, a margine di questo argomento, che anche per quanto riguarda la storia dei partiti in senso stretto il ricorso alle fonti locali è stato inopportunamente sottostimato e decisamente poco praticato. In secondo luogo, occorre notare come dopo il 1970 (ma in realtà fin dal dibattito costituente) la regione non sia solo un dominio di analisi, ma un fattore storico significativo nelle dinamiche del sistema. Questo per almeno due motivi. Da un lato perché, teoricamente, si propone non solo come un livello amministrativo tra gli altri, ma come base di un vero e proprio ripensamento federale dello Stato; e, come tale, delinea anche una prospettiva di riorganizzazione dei partiti stessi. Dall'altro perché, sul piano pratico, essa va ad alimentare ulteriormente la burocrazia pubblica e a costituire un nuovo e rilevante terreno 
di occupazione del potere e di lottizzazione partitica. Inoltre, sulla base delle sue competenze, essa diventa uno snodo fondamentale nella attuazione, ma anche nella crisi del welfare all'italiana.

La prospettiva di analisi regionale ci appare tanto più utile nella misura in cui focalizza qui un contesto che non rappresenta un semplice caso fra gli altri, ma un quadro di sostanziale differenza, quando non di speculare simmetria, rispetto al sistema nazionale. Anche in Emilia Romagna infatti ci troviamo di fronte un sistema politicamente vincolato (seppur in mano a quella che a livello nazionale è l'opposizione), con i relativi pericoli di immobilismo decisionale e tentazioni consociative; ma anche con peculiari virtù, in termini di sviluppo e coesione, che l'analisi storica ha variabilmente ricondotto alle tradizioni civiche, alla subcultura politica, all'industria diffusa. Occorre del resto rilevare come la periodizzazione della crisi del sistema politico nazionale appaia per molti versi parallela a quella del cosiddetto "modello emiliano", evidenziandone quindi i legami non secondari col quadro nazionale, troppo spesso trascurati in nome di una eccezionalità variamente connotata.

Ci proponiamo quindi di indagare la fase di crisi del sistema dei partiti nel contesto emiliano-romagnolo, secondo una prospettiva di storia politica intesa in senso ampio, capace di collegare la storia elettorale con quella amministrativa e le pratiche con le rappresentazioni. Dal punto di vista delle fonti, dando particolare rilievo, oltre alle carte dei partiti, a quelle degli enti locali, ma anche alle fonti statistiche, orali e iconografiche; sempre con una peculiare attenzione ai fondi locali. Ne deriva, già a un primo sguardo, un quadro mosso, nel quale il Pci emiliano acquisisce ulteriori sfumature, mentre gli altri soggetti ritrovano un ruolo di rilievo e la loro interazione si mostra tutt'altro che statica e lineare. La speranza è che con questi nuovi sguardi, e con le osservazioni che ne scaturiranno, si possa fornire un contributo non marginale alla storicizzazione del nostro presente e del nostro territorio.

A conclusione del dossier è stata organizzato, il 4 dicembre 2013, un seminario presso il Dipartimento di Storia, Culture Civiltà dell'Università di Bologna. Al dibattito, seguito all'introduzione di Alberto De Bernardi e agli interventi di Lorenzo Bertucelli, Marco Gervasoni e Marica Tolomelli, hanno partecipato gli autori Claudia Capelli, Mirco Carrattieri, Sante Cruciani, Carlo De Maria, Gisella Gaspari, Matteo Pasetti, Marianna Pino, Franco Piro, Gregorio Sorgonà, Matteo Troilo. I videoatti del seminario sono interamente disponibili sul web all'indirizzo: 䀧 www.youtube.com/playlist?list=PLPAaA-4eXcWz7pq-Fv7UXPIoH_ $\mathrm{VpL9nBq}$. 


\section{Bibliografia}

Asor Rosa A. 1996, La sinistra alla prova. Considerazioni sul ventennio 1976-1996, Torino: Einaudi

Baldissara L. (ed.) 2001, Le radici della crisi. L'Italia tra gli anni Sessanta e Settanta, Roma: Carocci

Barbagallo F. 2009, L'Italia repubblicana. Dallo sviluppo alle riforme mancate (19452008), Roma: Carocci

Bartolini S. e D’Alimonte R. (eds.) 2002, Maggioritario finalmente? La transizione elettorale 1994-2001, Bologna: il Mulino

Berselli E. 2003, Post-italiani. Cronache di un paese provvisorio, Milano: Arnoldo Mondadori

Cafagna L. 1993, La grande slavina. L'Italia verso la crisi della democrazia, Venezia: Marsilio

Calise M. 2006, La terza Repubblica. Partiti contro presidenti, Roma-Bari: Laterza

Caracciolo L. 1999, L'Italia alla ricerca di se stessa, in Sabbatucci G. e Vidotto V. (eds.) 1999, Storia d'Italia, VI, L'Italia contemporanea. Dal 1963 a oggi, Roma-Bari: Laterza

Casula C.F. (ed.) 1999, L'Italia dopo la grande trasformazione. Trent'anni di analisi Censis (1966-1996), Roma: Carocci

Ceccanti S. e Vassallo S. (eds.) 2004, Come chiudere la transizione. Cambiamento, apprendimento e adattamento nel sistema politico italiano, Bologna: il Mulino

Colarizi S. 1994, Storia dei partiti nell'Italia repubblicana, Roma-Bari: Laterza

Colarizi S. 2007, Storia politica della Repubblica. Partiti, movimenti e istituzioni, 19432006, Roma-Bari: Laterza

Crainz G. 2012, Il paese reale. Dall'assassinio di Moro all'Italia di oggi, Roma: Donzelli

Craveri P. 1995, La Repubblica dal 1958 al 1992, Torino: Utet

Craveri P. 2002, La democrazia incompiuta. Figure del Novecento italiano, Venezia: Marsilio

Craveri P. 2004, Dopo l'unità nazionale: la crisi del sistema dei partiti, in Colarizi S. et al. (eds.) 2004, Gli anni Ottanta come storia, Soveria Mannelli: Rubbettino

Deaglio E. 2009, Patria 1978-2008, Milano: Il Saggiatore

De Felice F. 2003, L'Italia repubblicana. Nazione e sviluppo, nazione e crisi, a cura di Masella L., Torino: Einaudi

De Felice R. 1995, Rosso e nero, Milano: Baldini\&Castoldi

De Luna G. 2011, La Repubblica del dolore. Le memorie di un'Italia divisa, Milano: Feltrinelli

Di Nolfo E. 1996, La Repubblica delle speranze e degli inganni. L'Italia dalla caduta del fascismo al crollo della Democrazia cristiana, Firenze: Ponte alle Grazie 
Flores d'Arcais P. 2011, Macerie. Ascesa e declino di un regime, 1986-2011, Reggio Emilia: Aliberti

Fruncillo D. 2004, Urna del silenzio. L'astensionismo elettorale in Italia, Roma: Ediesse Galli della Loggia E. 1996, La morte della patria, Roma-Bari: Laterza

Gentiloni Silveri U. 2008, Sistema politico e contesto internazionale nell'Italia repubblicana, Roma: Carocci

Gervasoni M. 2010, Storia d'Italia degli anni Ottanta. Quando eravamo moderni, Venezia: Marsilio

Ginsborg P. 1998, L'Italia del tempo presente. Famiglia, società civile, Stato 1980-1996, Torino: Einaudi

Giovagnoli A. (ed.) 1998, Interpretazioni della Repubblica, Bologna: il Mulino

Giovagnoli A. 2005, Il caso Moro: una tragedia repubblicana, Bologna: il Mulino

Gozzini G. 2011, La mutazione individualista. Gli italiani e la televisione 1954-2011, Roma-Bari: Laterza

Lanaro S. 1992, Storia dell'Italia repubblicana, Venezia: Marsilio

Losurdo D. 1994, La Seconda Repubblica. Liberismo, federalismo, postfascismo, Torino: Bollati Boringhieri

Lupo S. 1994, Il crepuscolo della Repubblica, in Lezioni sull'Italia repubblicana, Roma: Donzelli

Lupo S. 2013, Antipartiti, Roma: Donzelli

Mammarella G. 1993, L'Italia contemporanea, Bologna: il Mulino

Mastropaolo A. 1996, La Repubblica dei destini incrociati. Saggio su cinquant'anni di democrazia in Italia, Firenze: La Nuova Italia

Morlino L. e Tarchi M. (eds.) 2006, Partiti e caso italiano, Bologna: il Mulino

Neppi Modona G. (ed.) 1996, Cinquant'anni di Repubblica italiana, Torino: Einaudi

Pezzino P. 2002, Senza Stato. Le radici storiche della crisi italiana, Roma-Bari: Laterza

Quagliariello G. 2004, Gli anni Ottanta: gli aspetti istituzionali. Un'interpretazione, in Colarizi S. et al. (eds.) 2004, Gli anni Ottanta come storia, Soveria Mannelli: Rubbettino

Ridolfi M. 2010, Storia politica dell'Italia repubblicana, Milano: Bruno Mondadori

Sabbatucci G. 2003, Il trasformismo come sistema, Roma-Bari: Laterza

Salvadori M. 1994, Storia d'Italia e crisi di regime. Alle radici della politica italiana, Bologna: il Mulino

Salvati M. 2000, Occasioni mancate. Economia e politica in Italia dagli anni '60 a oggi, Roma-Bari: Laterza

Santarelli E. 1996, Storia critica della Repubblica. L'Italia dal 1945 al 1994, Milano: Feltrinelli 
Scoppola P. 1991, La repubblica dei partiti, Bologna: il Mulino

Sylos Labini P. 1995, La crisi italiana, Roma-Bari: Laterza

Tranfaglia N. 1994, L'Italia democratica. Profilo del primo cinquantennio 1943-1994, Milano: Unicopli

Tranfaglia N. 2009, Vent'anni con Berlusconi (1993-2013). L'estinzione della sinistra, Milano: Garzanti

Tullio-Altan C. 1986, La nostra Italia, Milano: Feltrinelli

Vassallo S. 1994, Il governo di partito in Italia (1943-1993), Bologna: il Mulino

Ventrone A. (ed.) 2006, L'ossessione del nemico. Memorie divise nella storia della Repubblica, Roma: Donzelli

Zavoli S. 1992, La notte della Repubblica, Milano: Arnoldo Mondadori

Zavoli S. 1999, C'era una volta la prima Repubblica, Milano: Arnoldo Mondadori 